\title{
Xenobiotic Metabolism in Alginate-Encapsulated Primary Human Hepatocytes Over Long Timeframes
}

\author{
Martin B. Phillips, ${ }^{1}$ Pergentino Balbuena-Venancio,,2 Jeffrey R. Enders, ${ }^{1,}$ Rachel L. Norini, \\ Yoo-Sik Shim, ${ }^{1,2,{ }^{*}}$ Erin Burgunder, ${ }^{1,2, \dagger}$ Lavanya Rao,' David Billings, ${ }^{1,2}$ Jenny Pedersen, \\ Jeffrey M. Macdonald, Melvin Andersen, ${ }^{1,2}$ Harvey J. Clewell, III, ${ }^{1,2, \$}$ and Miyoung Yoon ${ }^{1,2, \$}$
}

\begin{abstract}
Introduction: Application of in vitro-to-in vivo extrapolation to chemical safety assessment has gained significant attention in the past few years. Accurate prediction of hepatic metabolic clearance from in vitro data is critical in this regard as it determines compound pharmacokinetics and exposure at sites of action. To estimate hepatic metabolic clearance, intrinsic clearance $\left(\mathrm{CL}_{\mathrm{int}}\right)$ is typically measured using in vitro systems such as primary hepatocytes or subcellular fractions derived from liver tissues. However, currently available in vitro systems used in these studies often lack long-term metabolic competence, making it challenging to estimate $\mathrm{CL}_{\text {int }}$ for poorly metabolized and slowly cleared chemicals.

Materials and Methods: To address this challenge, we developed a three-dimensional (3D) primary human hepatocyte cell culture system using alginate hydrogels ("alginate bead culture") with extended viability and metabolic competence.

Results: Alginate bead culture generates comparable estimates of metabolism to other in vitro and in vivo systems. In addition, expression of several xenobiotic metabolizing enzymes over long timeframes in beads is comparable to freshly isolated hepatocytes in suspension culture.

Discussion: We compare $\mathrm{CL}_{\text {int }}$ estimated from traditional suspension versus alginate bead culture for two chemicals ( $S$-warfarin and coumarin) and show that alginate bead culture holds promise to increase confidence in estimates of $\mathrm{CL}_{\text {int }}$ for slowly cleared chemicals.

Conclusions: In addition to providing an improved in vitro tool to estimate metabolic clearance for a broad range of chemicals, this simple 3D hepatocyte culture can be adapted to a continuous flow-based bioreactor system allowing repeated exposure experiments over long periods in vitro, tackling an important challenge in in vitrobased chemical safety assessment.
\end{abstract}

Keywords: 3D culture, hepatocytes, metabolism, slow clearance

\section{Introduction}

I N AN EFFORT TO MODERNIZE TOXICITY testing to improve its efficiency and human relevance, new in vitro assays have been developed to determine the biological activities of chemicals. ${ }^{1}$ To put the results of these tests into their appropriate context and predict potential impacts to human health, it is crucial to account for pharmacokinetics as in vitro assays inher- ently lack absorption, distribution, metabolism, and excretion (ADME) processes. ADME parameters can be determined using in vitro assays, which can then be extrapolated to the corresponding in vivo parameters. ${ }^{2-5}$ This biological scaling process is referred as in vitro-to-in vivo extrapolation (IVIVE). Accurate prediction of metabolism, particularly hepatic metabolism as the liver is the major site of metabolism, is important because metabolism often dominates overall

\footnotetext{
${ }^{1}$ ScitoVation, LLC, Research Triangle Park, North Carolina.

${ }^{2}$ Institute for Chemical Safety Sciences, The Hamner Institutes for Health Sciences, Research Triangle Park, North Carolina.

${ }^{3}$ Joint Department of Biomedical Engineering, University of North Carolina, Chapel Hill, North Carolina.

*Current affiliation: University of California, San Diego, California.

Current affiliation: University of North Carolina, Chapel Hill, North Carolina.

Current affiliation: Ramboll, Research Triangle Park, North Carolina.

${ }^{\S}$ Current affiliation: ToxStrategies, Inc., Research Triangle Park, North Carolina.
}

( ) Martin B. Phillips et al., 2018; Published by Mary Ann Liebert, Inc. This Open Access article is distributed under the terms of the Creative Commons License (http://creativecommons.org/licenses/by/4.0), which permits unrestricted use, distribution, and reproduction in any medium, provided the original work is properly cited. 
clearance of a compound along with the processes of bioactivation and detoxification, in turn determining internal exposure of bioactive compounds at the target tissues.

Several in vitro systems are available for metabolism studies ranging from expressed enzymes, liver subcellular fractions, primary hepatocytes, and other cell-based assays. ${ }^{6}$ For chemical safety assessment, it would be ideal to conduct full enzyme kinetic studies and determine $V_{\max }$ and $K_{\mathrm{m}}$ for two major reasons; (1) the metabolites are frequently a concern for toxicity and (2) metabolic saturation may occur at high doses such as in animal toxicity studies requiring measurement of both capacity $\left(V_{\max }\right)$ and affinity $\left(K_{\mathrm{m}}\right)$.

In practice, intrinsic clearance $\left(\mathrm{CL}_{\mathrm{int}}\right)$ can be used to estimate in vivo hepatic metabolic clearance since metabolic processes are not likely to be saturated at typical human exposure levels. ${ }^{7}$ As it is more amenable to high-throughput screening than the traditional enzyme kinetic assays, the $\mathrm{CL}_{\text {int }}$ approach has been applied to high-throughput dosimetry studies for margin of exposure analysis based on high-throughput in vitro bioactivity screening data and human exposure estimates. ${ }^{8,9}$ This approach, however, is only applicable if the parent compound is responsible for bioactivity. When metabolites contribute to toxicity, other factors, including rates of formation and clearance of the metabolite in addition to the parent compound, would be necessary to estimate in vivo exposures to all the active entities.

The use of $\mathrm{CL}_{\text {int }}$ in IVIVE has shown its utility in predicting in vivo hepatic clearance for drug compounds if nonspecific binding in the system is appropriately addressed and unbound or free substrate concentrations are used for IVIVE. ${ }^{10-13}$ One limitation of applying this approach to environmental chemicals, however, is that available in vitro incubation methods commonly used for $\mathrm{CL}_{\text {int }}$ determination are largely limited to rapidly metabolized and/or relatively water soluble chemicals. For most in vitro preparations, incubations are restricted to short exposure periods, making it a challenge to accurately determine $\mathrm{CL}_{\text {int }}$ for slowly metabolized chemicals and/or highly<smiles>CC(=O)C[C@H](c1ccccc1)c1c(O)c2ccccc2oc1=O</smiles>

S-Warfarin<smiles>CC(=O)CC(c1ccccc1)c1c(O)c2ccc(O)cc2oc1=O</smiles>

7-Hydroxywarfarin

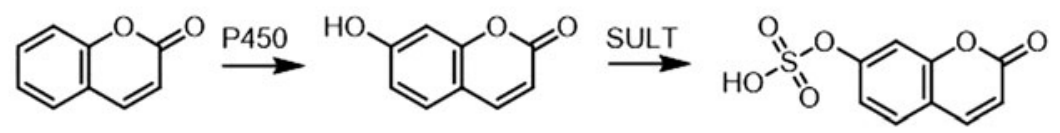

Coumarin
7-Hydroxycoumarin

7-Hydroxycoumarin glucuronide

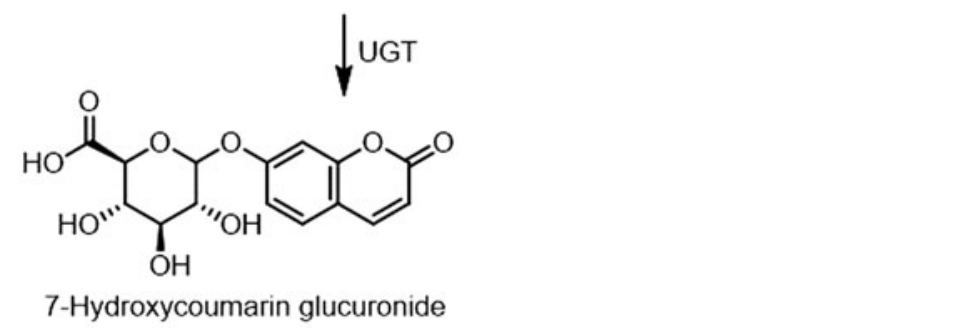

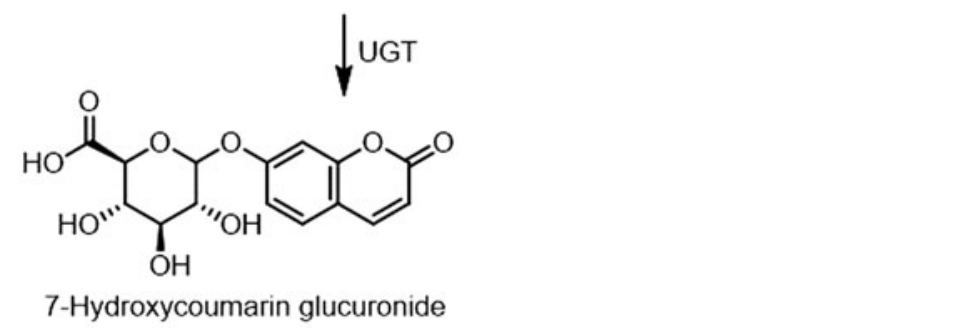

lipophilic chemicals. This deficiency represents a major source of uncertainty for estimating $\mathrm{CL}_{\text {int }}$ for IVIVE to support safety assessment.

The best in vitro predictions for rapidly cleared chemicals use freshly isolated or cryopreserved primary hepatocytes in suspension. Use of monolayer or sandwich-culture of hepatocytes extends viability but not necessarily their metabolic competence. With most primary hepatocyte-based culture systems, there is clear phenotypic instability and rapid decline of metabolic competence within a few hours, making them unsuitable for long-term incubations. ${ }^{13,14}$

Several techniques can stabilize hepatic phenotype over longer periods and show promise for enhancing the predictive power of primary cell culture with respect to estimating in vivo pharmacokinetics. ${ }^{15,16}$ Advanced cell culture models such as scaffold-based three-dimensional (3D) cultures, spheroid cultures, or 3D encapsulated spheroids of primary human hepatocytes, such as the one we optimized in this study, maintain hepatocyte morphology and viability over many weeks. ${ }^{17,18}$

Among these models, we selected alginate hydrogel-based $3 \mathrm{D}$ cultures for estimating $\mathrm{CL}_{\text {int }}$ for low clearance chemicals. These preparations have greater scalability compared to scaffold-based or spheroid culture (i.e., the number of beads can be increased or decreased based on experimental needs); they are relatively easy to model computationally to extract in vitro kinetic parameters; they have long-term stability, from many hours to weeks; and they can avoid the necrotic core issues frequently seen in 3D hepatocyte cultures.

The objective of this study was to evaluate the utility of alginate bead culture for long-term primary human hepatocyte culture model with slowly metabolized chemicals. $S$-Warfarin and coumarin (Fig. 1) were selected as case compounds with different $C L_{\text {int }}$ ranges to examine the degree of metabolic competence of the hepatocytes cultured in alginate bead culture over time. To verify metabolic competence of alginate bead culture reflected in $\mathrm{CL}_{\text {int }}$ of those test compounds, expression of a few selected Phase I and Phase II xenobiotic metabolizing enzymes (XMEs) was measured over
FIG. 1. Chemical structures of $S$-warfarin (left) and coumarin and its metabolites (right). 
time, with results normalized to expression in freshly isolated cells from the same donor.

\section{Materials and Methods}

\section{Materials}

Hepatocytes derived from seven donors were used: three for gene expression analysis, one for alginate bead culture only, and three for both alginate bead and suspension cultures. LHuf15906 was used for expression analysis; the donor was a 28-year-old Caucasian female with a body mass index (BMI) of 25.7, a history of alcohol and tobacco, but not other drug use, and negative serologies (HIV, HBV, and HCV). HUM4179 was also used for expression analysis; the donor was a 27 -year-old Hispanic male with a BMI of 26.7, a history of tobacco, marijuana, and cocaine use, and negative serologies. HUM4108 was also used for expression analysis; the donor was a 42-year-old African American female with a BMI of 35, a history of alcohol, but not tobacco or other drug use, and negative serologies. HUM4197 was used for alginate bead culture; the donor was a 14-year-old Caucasian male with a BMI of 22, no history of alcohol, tobacco, or drug use, and negative serologies. Hu161112, HUM4209, and HUM4216 were used for both alginate bead and suspension cultures; the donor Hu161112 was a 10-month-old Caucasian female. The donor HUM4209 was a 60 -year-old Caucasian female with a BMI of 25, a history of alcohol and tobacco, but not other drug use, and negative serologies. The donor HUM4216 was an 8-year-old Hispanic female with negative serologies.

Freshly isolated primary human hepatocytes were purchased from Lonza (Research Triangle Park, NC), with the exception of LHuf15906, which was purchased from LifeNet. William's E medium, Hank's balanced salt solution (HBSS), 4-(2-hydroxyethyl)-1-piperazineethanesulfonic acid (HEPES), Glutamax, and penicillin/streptomycin were purchased from Life Technologies (Grand Island, NY). HEPES (powder), sodium chloride $(\mathrm{NaCl})$, potassium chloride $(\mathrm{KCl})$, calcium chloride $\left(\mathrm{CaCl}_{2}\right)$, alginic acid sodium salt from brown algae (low viscosity, powder form), dexamethasone, $(S)-(-)$-warfarin, coumarin, 7-hydroxycoumarin, 7-hydroxycoumarin sulfate, and 7-hydroxycoumarin glucuronide were purchased from Sigma-Aldrich (St. Louis, MO).

7-Ethoxycoumarin- $d_{5},(R S)-( \pm)$-warfarin, warfarin- $d_{5}, 4^{\prime}$ hydroxywarfarin, and $4^{\prime}$-hydroxywarfarin- $d_{4}$ were purchased from Toronto Research Chemicals (Toronto, ON). Acridine orange (AO)/propidium iodide (PI) staining solution was purchased from Nexcelom Bioscience (Lawrence, MA). ITS+ Premix was purchased from Corning (Corning, NY). TaqMan Universal Master Mix II without uracil $N$-glycoslyase and cell culture plates were purchased from ThermoFisher (Waltham, MA). RNeasy Micro Kits were purchased from Qiagen (Venlo, Netherlands).

Calcium crosslinking solution for use with the encapsulator consisted of $10 \mathrm{mM}$ HEPES, $142 \mathrm{mM} \mathrm{NaCl}, 6.7 \mathrm{mM} \mathrm{KCl}$, and $200 \mathrm{mM} \mathrm{CaCl}_{2}$ in distilled water. The $\mathrm{pH}$ of the crosslinking solution was adjusted to 7.4 using sodium hydroxide $(\mathrm{NaOH})$, then filter sterilized with a $0.22 \mu \mathrm{m}$ polyethersulfone filter. Hepatocyte maintenance media consisted of William's E medium supplemented with insulin $(10 \mu \mathrm{g} / \mathrm{mL})$, transferrin $(5.5 \mu \mathrm{g} / \mathrm{mL})$, selenium $(5 \mathrm{ng} / \mathrm{mL})$, dexamethasone $(0.1 \mu \mathrm{M})$, penicillin $(100 \mathrm{U} / \mathrm{mL})$, streptomycin $(100 \mu \mathrm{g} / \mathrm{mL})$, and Glutamax $(10 \%[\mathrm{v} / \mathrm{v}])$.

\section{Methods}

Suspension culture. Freshly isolated human hepatocytes (Hu161112) were added to a 24-well cell culture plate (1.3 million cells/well, $1 \mathrm{~mL}$ total volume/well) and cultured under dynamic conditions at $37^{\circ} \mathrm{C}$ and $5 \% \mathrm{CO}_{2}$ in hepatocyte maintenance media on a rotating platform at $120 \mathrm{rpm}$ (VWR 3500 Advanced Digital Orbital Shaker Mixer, Radnor, PA).

Hepatocytes were received on ice immediately after isolation from the vendor who was located in the same building and were warmed in the incubator at $37^{\circ} \mathrm{C}$ and $5 \% \mathrm{CO}_{2}$ before compound treatment ( $<5$ minutes). Hepatocytes were treated with coumarin at $1,5,10$, or $50 \mu \mathrm{M}$ or with warfarin at $0.1,0.5,1$, or $5 \mu \mathrm{M}$. Each concentration was run in duplicate. Every hour for 4 hours, $50 \mu \mathrm{L}$ media was collected and stored at $-80^{\circ} \mathrm{C}$ for analysis.

\section{Dynamic 3D culture}

Encapsulation. Before encapsulation, cells were counted on a hemocytometer, using Trypan Blue to assess viability. Freshly isolated human hepatocytes (HUM4197) were centrifuged at $100 \mathrm{~g}$ and mixed with $2 \%(\mathrm{w} / \mathrm{v})$ alginate in HBSS at a $1: 1.25$ ratio $(\mathrm{v} / \mathrm{v})$ of cells to $2 \%(\mathrm{w} / \mathrm{v})$ alginate. The mixture was fed at $14 \mathrm{~mL} / \mathrm{h}$ using a syringe pump through the encapsulator at $5.5 \mathrm{kV}$, using a 24-gauge needle, into a continuously stirred container of calcium crosslinking solution described above, to form alginate beads $\sim 500 \mu \mathrm{m}$ in diameter. "Blank beads" were formed using alginate alone, without cells. A Var V1 encapsulation unit was used for all encapsulation process (Nisco Engineering AG, Zürich, Switzerland).

After encapsulation, the crosslinking solution was replaced with a volume of fresh hepatocyte maintenance media and kept under stirring conditions using a magnetic stir bar, creating a well-mixed dispersion of beads and a known concentration of cells in media. Using this concentration, a volume of beads in media was distributed to each well to achieve the target number of cells/well. Using an average volume of a human hepatocyte of $11 \mathrm{pL}$ (based on a cell diameter of $27.6 \mu \mathrm{m})$ and the $1: 1.25$ volume ratio of cell pellet to alginate, the cell density in an alginate bead was calculated. Using bead size distribution and cell density in an alginate bead, the number of beads in each well was calculated ( $\sim 1000$ beads per well).

Compound treatment. The alginate beads were added to a six-well cell culture plate $(4$ million cells/well and $3 \mathrm{~mL}$ total volume/well) and cultured under dynamic conditions at $37^{\circ} \mathrm{C}$ and $5 \% \mathrm{CO}_{2}$ in hepatocyte maintenance media on a rotating platform at $120 \mathrm{rpm}$. Beads were preincubated after encapsulation before compound treatment $(<5 \mathrm{~min}-$ utes). Alginate beads were treated at an initial concentration of $0.1,0.5,1,5$, or $10 \mu \mathrm{M} S$-warfarin or 10,50 , or $100 \mu \mathrm{M}$ of coumarin (day 0). Each concentration was run in duplicate. Every 24 hours for 7 days, $1 \mathrm{~mL}$ media was collected and stored at $-80^{\circ} \mathrm{C}$ for analysis, and replaced with $1 \mathrm{~mL}$ fresh hepatocyte maintenance media.

Additional controls were run in a similar manner to the alginate beads. "Blank bead" controls were run with alginate beads prepared without cells. A similar number of beads were added to each well as in the hepatocyte-containing preparations. "Media-Only" controls were run with $3 \mathrm{~mL}$ of media added to each well without any beads. Control 
wells were otherwise handled identically, with daily media changes and dosing on day 0 .

$X M E$ expression analysis. Alginate beads prepared using freshly isolated primary human hepatocytes were cultured in six-well plates (6 million cells/well, $3 \mathrm{~mL}$ total volume/ well) and the expression of selected XME mRNA was measured using quantitative reverse transcription-polymerase chain reaction (qRT-PCR). RNeasy Micro Kits were used to purify the RNA from the beads, and TaqMan Universal Master Mix II without uracil $N$-glycoslyase was used for the qRTPCR steps. The XMEs selected for this study were CYP1A2, CYP2C9, CYP2D6, and CYP2E1 (cytochrome P450s; Phase I oxidative metabolism), CES1 (carboxylesterase; Phase I non-CYP metabolism), UGT2B7 (uridine 5'-diphospho-glucuronosyltransferase; Phase II conjugation), and SULT1A1 (sulfotransferase; Phase II conjugation).

For the first donor (LHuf15906), expression was measured on day 0 (the day of encapsulation) pre- and postencapsulation. For the second donor (HUM4179), beads from six wells ( $\sim 125,000$ cells, $63 \mu \mathrm{L}$ ) were harvested on day 3 for expression analysis, and beads from three of those six wells were harvested again on day 7 for analysis. For the third donor (HUM4108), expression was measured on day 0 postencapsulation. One independent well was harvested on each of days 7 , 17, 21, and 28, and expression was measured. All qRT-PCR results were normalized to a housekeeping gene (B2M) and then normalized again to the day 0 expression value for each gene.

Staining, imaging, and measurement of size distribution of alginate beads. Alginate beads were stained with AO/PI from Nexcelom Biosciences, and imaged using a Nikon Eclipse T-100 inverted fluorescent scope with Nikon objectives. Alginate bead size was measured using Nikon's NIS-Elements D software v. 4.30.02. The size distribution of bead diameters using our encapsulation method was $750 \pm 173 \mu \mathrm{m}$ (range $440-1100 \mu \mathrm{m})$.

Liquid chromatography-mass spectrometry methods. Two liquid chromatography-mass spectrometry (LC-MS) methods were used: one for coumarin and associated metabolites and the other for warfarin and an associated metabolite. Reference standards were diluted to appropriate calibrator level concentrations $(0.2,0.5,1,5,25,100,250,1000,5000 \mathrm{ng}$ analyte $/ \mathrm{mL}$ cell culture media) and were then diluted an additional $10 \times$ by taking $100 \mu \mathrm{L}$ of calibrator and adding it to $900 \mu \mathrm{L}$ of internal standard (7-ethoxycoumarin- $d_{5}$ at $500 \mathrm{ng} / \mathrm{mL}$, dissolved in deionized water or warfarin- $d_{5} / 4^{\prime}$-hydroxywarfarin- $d_{4}$ at $500 \mathrm{ng} / \mathrm{mL}$, dissolved in deionized water). No sample cleanup/ extraction was performed other than dilution as these cleanup steps affected metabolite detection. Cellular media samples were treated similarly: $10 \mu \mathrm{L}$ of media sample was mixed with $90 \mu \mathrm{L}$ of one of the aqueous internal standard solutions (depending on which assay was being run) and vortexed.

All mass spectrometric analyses were conducted by LC-MS/ MS on an Agilent 6460 platform run in electrospray ionization mode using an Agilent 1290 chromatographic system (1290 Infinity Binary Pump, 1290 TCC, 1290 Sampler and 1290 Thermostat) with a Phenomenex SecurityGuard ULTRA Cartridge UHPLC Fully Porous C18 for $2.1 \mathrm{~mm}$ ID Columns (AJ0-9502) followed by a Phenomenex Luna Omega $1.6 \mu \mathrm{m}$
C18 $100 \AA$ LC Column 50×2.1 mm (00B-4742-AN) analytical column. Methods for warfarin and coumarin used the same chromatographic setup. The run time for the coumarin method is 8 minutes, and for the warfarin method is 5 minutes. These assays monitor at least two transitions for all analytes and internal standards. Mass spectrometry method parameters are summarized in Supplementary Table S1 (Supplementary Data are available online at www.liebertpub.com/aivt).

LC-MS methods were validated using a variety of metrics, including limits/sensitivity (the lower and upper concentration limits at which the method could accurately identify and quantify the analyte), linearity (the reproducible regression or fit of the calibration curve), carryover (the highest concentration of analyte present which does not produce a concentration level above the lower limit in a blank injection proceeding it), precision and accuracy (the capability of the method to produce the reproducible accurate results over a period of multiple days at concentrations spanning the concentration range of interest), interference/selectivity (the ability of the method to be unaffected by the presence of other medications/compounds), and matrix effects (the suppression or enhancement of analyte signals of interest due to the presence of matrix -in this case cellular media). A thorough explanation of this validation process may be found elsewhere. ${ }^{19}$

Computational modeling. Computational modeling of the in vitro kinetics in the system was performed in MATLAB (Natick, MA). The code used to analyze the time course data is in the Supplementary Data. This code estimates pseudo-first order rate constants $\left(\right.$ day $\left.^{-1}\right)$ in the in vitro system. To convert these values to in vivo human clearance, IVIVE was used. ${ }^{12}$ The hepatocellularity per gram liver used as a scaling factor for IVIVE is $99 \times 10^{6}$ cells/g. ${ }^{20}$ The liver weight per $\mathrm{kg}$ body weight (BW) used in this IVIVE is $26 \mathrm{~g} .{ }^{21}$ See Equation (1) for how to calculate in vivo $\mathrm{CL}_{\text {int }}$ (in units of $\mathrm{mL} / \mathrm{h} / \mathrm{kg} \mathrm{BW}$ ).

$$
\begin{aligned}
C L_{\text {int }}=k\left(\text { day }^{-1}\right) \cdot \frac{1 \text { day }}{24 h} \cdot \frac{\text { Volume }(\mathrm{mL}) \text { incubation }}{4 \times 10^{6} \text { cells }} . \\
\frac{99 \times 10^{6} \text { cells }}{g \text { liver }} \cdot \frac{26 \mathrm{~g} \text { liver }}{\mathrm{kg} \mathrm{BW}}
\end{aligned}
$$

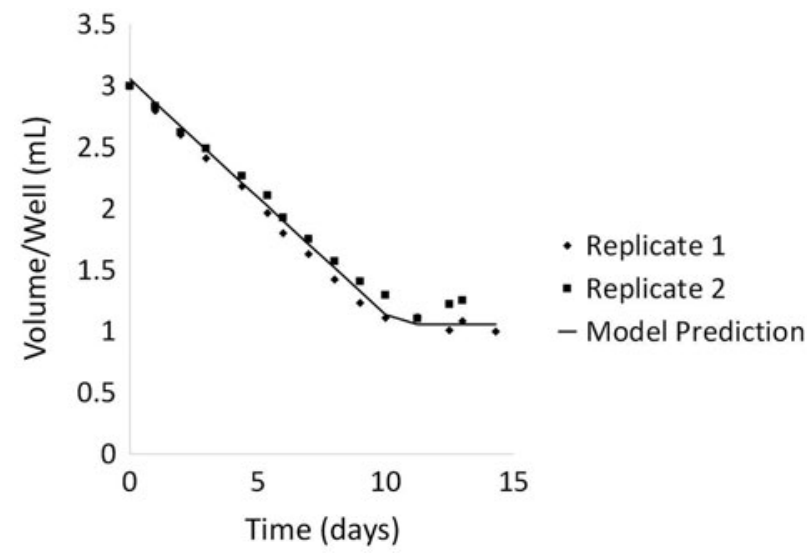

FIG. 2. Volume per well versus time. Experimental data are shown as points, the model prediction using Equation (2) is shown as a solid line. 
The model also includes changes in the volume of media per well due to evaporation during 7-day incubation period. Because $1 \mathrm{~mL}$ of media is removed during sampling and replaced with the same volume of fresh media each day, the volume per well could not drop below this limit. A sixwell plate containing only media $(3 \mathrm{~mL} /$ well $)$ was placed in the cell culture incubator to test for evaporation. Every 24 hours, $1 \mathrm{~mL}$ was removed, $1 \mathrm{~mL}$ of fresh media was added, and the plate was weighed. Equation (2) shows the best fitting model for evaporation from the plate ( $t$ is time in days).

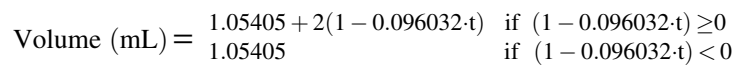

The fit of Equation (2) to the evaporation plate data is shown in Figure 2.
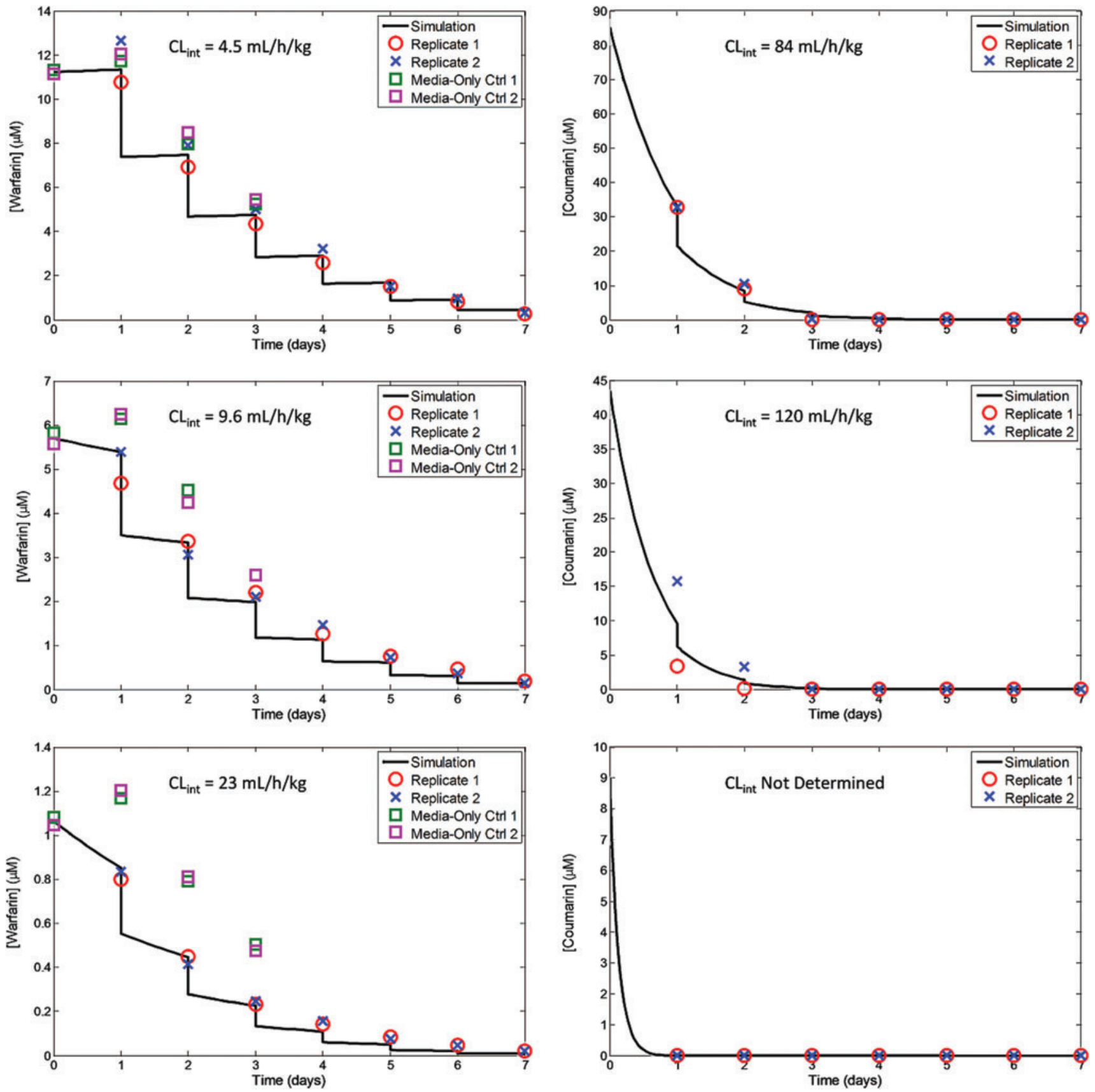

FIG. 3. Warfarin (left) and coumarin (right) time course data for donor HUM4197. The initial nominal concentration of warfarin in the media at day 0 was $10 \mu \mathrm{M}$ (top), $5 \mu \mathrm{M}$ (middle), or $1 \mu \mathrm{M}$ (bottom). The initial nominal concentration of coumarin in the media at day 0 was $100 \mu \mathrm{M}$ (top), $50 \mu \mathrm{M}$ (middle), or $10 \mu \mathrm{M}$ (bottom). Estimated in vivo $\mathrm{CL}_{\text {int }}$ values based on each in vitro concentration-time curve data are indicated in the plot. Replicates were from the same human donor. Mediaonly controls were wells with the same initial concentration of warfarin on day 0 ; changes in concentration in these controls over time reflect a combination of evaporation, which tends to concentrate the analyte, and dilution due to daily media changes. Color images available online at www.liebertpub.com/aivt 
Nonlinear kinetic analysis [Eq. (3)] was performed using the pseudo-first order rate constants $(k)$ derived from the time course data for substrate $(S)$ disappearance. Weighted nonlinear least squares regression was used to find the $k_{\text {dep }}([S] \rightarrow 0)$ and $K_{\mathrm{m}}$ values for each compound. Both $k_{\text {dep }}$ and $k_{\mathrm{dep}}([S] \rightarrow 0)$ have units of days ${ }^{-1}, K_{\mathrm{m}}$ and $[S]$ have units of $\mu \mathrm{M}^{22}$

$$
k_{d e p}=k_{d e p}([S] \rightarrow 0)\left(1-\frac{[s]}{[s]+K_{m}}\right)
$$

\section{Results}

\section{Evaporation study}

During the course of our incubations, the rate of evaporation was noted to be significant, so this rate was experimentally determined to include it in the computational model to appropriately correct media concentrations considering the gradual changes in volume for IVIVE. Despite the high humidity in the cell culture incubators, $\sim 100 \mu \mathrm{L}$ was lost to evaporation from each well each day. Over the course of a week-long study, this amounted to nearly $1 / 3$ of the total volume; thus, this parameter is important to characterize based on the actual conditions for the system. Our viability and XME expression experiments were conducted in the same incubators, so all data are comparable in our system.

\section{Time course studies}

Warfarin and coumarin were used to treat alginate bead cultures and suspension hepatocytes. Only freshly isolated primary human hepatocytes were used in this study; attempts at encapsulating cryopreserved hepatocytes were unsuccessful and were characterized by extreme loss of viability during the encapsulation process (unpublished results from our laboratory). Multiple concentrations for each compound were tested, and the time course for parent disappearance in respective culture media was measured using LC-MS.

The time course for warfarin disappearance in bead culture in one of the donors is shown in Figure 3, while all other results for warfarin and coumarin in bead and suspension culture are shown in Supplementary Data (Supplementary Figs. S1-S4). Note that media-only controls show changes in warfarin concentrations over time due to daily changes in media (dilution) and evaporation of the media (concentration). The experimental replicates show further decreases in concentrations, representing the amount of warfarin that was metabolized. The time course data were analyzed using a computational model as described in the "Methods" section and in vivo $\mathrm{CL}_{\text {int }}$ was estimated using IVIVE (Table 1). Warfarin disappearance shows the saturable nature of metabolism with an estimated $K_{\mathrm{m}}$ at $7.4 \mu \mathrm{M}$ and $k_{\mathrm{dep}}([S] \rightarrow 0)=0.76$ days $^{-1}$ (Supplementary Fig. S5). To estimate in vivo $\mathrm{CL}_{\mathrm{int}}$, only the concentrations below the estimated $K_{\mathrm{m}}$ were used (Table 1 ).

The estimated in vivo $\mathrm{CL}_{\text {int }}$ for warfarin based on alginate bead culture data was within fourfold of the reported in vivo data. ${ }^{23}$ For coumarin, the in vivo $\mathrm{CL}_{\mathrm{int}}$ estimated from the alginate beads shortly after encapsulation on day 0 was within threefold of the estimate using the data from hepatocyte suspension culture (Supplementary Table S2), indicating that the encapsulation process itself did not substantially affect the metabolic competence of the hepatocytes encapsulated
TABle 1. $S$-Warfarin Intrinsic Clearance $\left(\mathrm{CL}_{\text {INT }}, \mathrm{ML} / \mathrm{H} / \mathrm{KG}\right.$ BODY WEIGHT) ESTIMATES FROM IN VITRo VERSUS IN VIVO REPORTED DATA

In vivo $C L_{\text {int }}(\mathrm{mL} / \mathrm{h} / \mathrm{kg} \mathrm{BW})$ estimated from alginate bead incubations

\begin{tabular}{lcccc}
\hline $\begin{array}{l}\text { Substrate } \\
\text { concentration }\end{array}$ & $0.1 \mu M$ & $0.5 \mu M$ & $1 \mu M$ & Average $^{\mathrm{a}}$ \\
\hline $\begin{array}{l}\text { S-Warfarin } \mathrm{CL}_{\mathrm{int}}^{\mathrm{b}} \\
\text {-W } 8983\end{array}$ & $53 \pm 42$ & $52 \pm 27$ & 65 \\
\hline \begin{tabular}{l} 
In vivo $\mathrm{CL}_{\mathrm{int}}(\mathrm{mL} / \mathrm{h} / \mathrm{kg} \mathrm{BW})$ \\
\hline in Halifax et al.
\end{tabular} & 270 \\
\hline${ }^{\mathrm{a}}$ Average of the $\mathrm{CL}_{\text {int }}$ estimates from $0.1,0.5$ and $1 \mu \mathrm{M}$. \\
b Values are given \pm standard deviation for biological replicates \\
from three different donors for 0.1 and $0.5 \mu \mathrm{M}$ and from four differ- \\
ent donors for $1 \mu \mathrm{M}$. \\
BW, body weight.
\end{tabular}

into the beads. This result is also consistent with the observed similarity in XME expression levels and profiles in the hepatocytes before and after encapsulation. It should be noted that the calculated $\mathrm{CL}_{\mathrm{int}}$ for coumarin may have been underestimated as it may have been completely metabolized before the 24 hours sampling point.

\section{XME gene expression studies}

To characterize metabolic enzyme expression in the alginate bead culture over time, mRNA levels of genes encoding for several XMEs were quantitated using qRT-PCR in alginate bead hepatocytes derived from three human donors. First, the effects of the encapsulation process itself were investigated by comparing expression in freshly made beads on day 0 with suspension culture (Fig. 4).

Next, expression was tracked over timeframes relevant to the current study. XME expression in beads prepared using cells from the second human donor are shown in Figure 5, with AO/PI viability staining shown in Figure 6. Expression in alginate bead culture was normalized to expression in

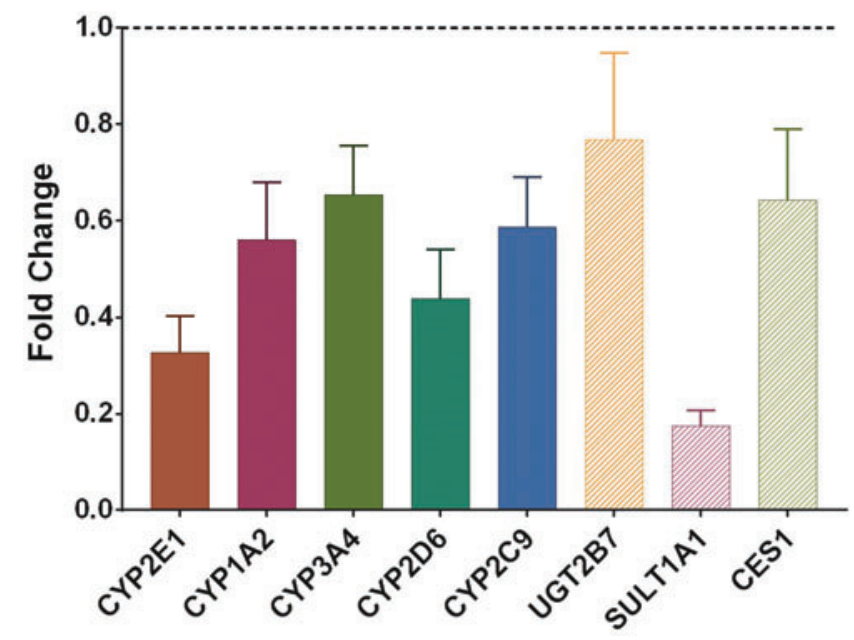

FIG. 4. Comparing pre- and postencapsulation gene expression for the first donor (LHuf15906). Error bars represent the standard deviation in qRT-PCR replicates $(n=3)$. qRT-PCR, quantitative reverse transcription-polymerase chain reaction. Color images available online at www.liebertpub.com/aivt 
FIG. 5. Gene expression for the second donor (HUM4179) over time normalized to expression in suspension culture on day 0 . Error bars represent variation between replicates ( $n$ for each time point is indicated on the x-axis). Color images available online at www liebertpub.com/aivt

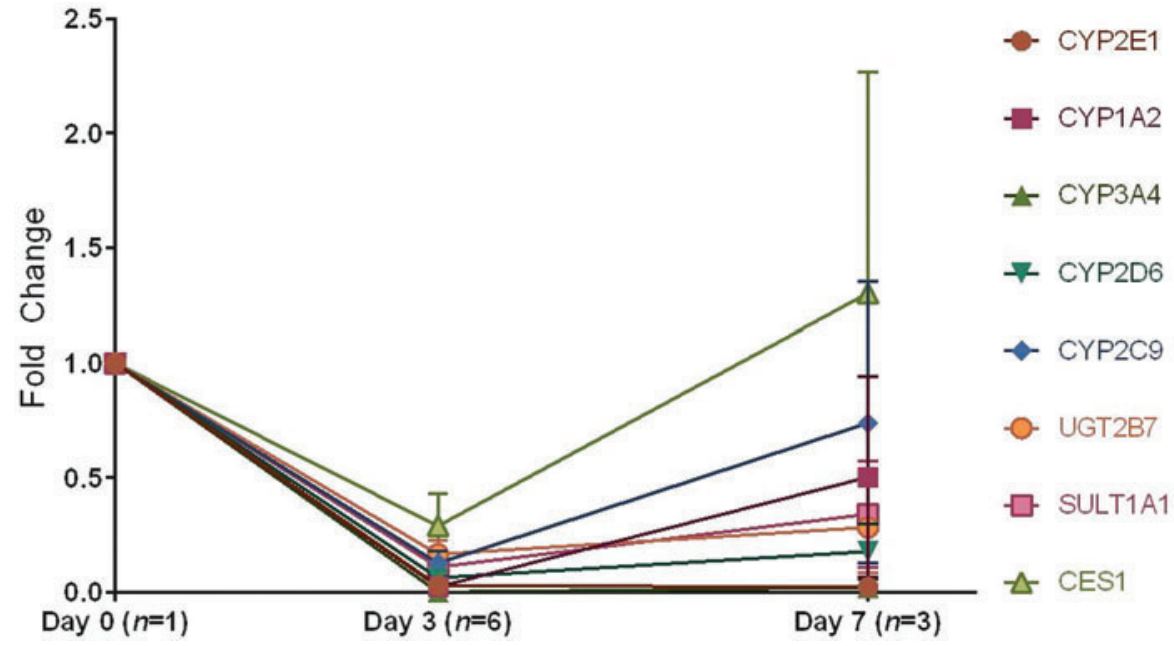

freshly isolated cells from the same donor in suspension culture. These results show a decrease in expression over the first 3 days of culture, followed some rebound between day 3 and 7 postencapsulation. Viability staining shows no major time-dependent changes over this period.

Expression for the third human donor was measured on day 28 postencapsulation and compared to beads on day 0 (Fig. 7). This shows that CYP1A2, CYP3A4, CYP2C9, SULT1A1, and CES1 were all expressed at or above day 0 levels, while CYP2E1, CYP2D6, and UGT2B7 were all expressed, although at relatively lower levels. AO/PI viability staining over the course of the 28 day study shows that the cells appeared relatively normal (Fig. 8).

\section{Discussion}

In a previous study, we showed that alginate bead cultures can extend hepatocyte viability to more than 4 weeks. ${ }^{24}$ Here, we further optimized the system and evaluated the metabolic competence of this simple 3D hepatocyte culture for determining $\mathrm{CL}_{\text {int }}$ for slowly cleared chemicals. Warfarin was chosen as a case study compound due to its slow metabolic clearance and the existence of in vivo data to use as a benchmark. ${ }^{23,25,26}$ Coumarin was chosen due to its structural similarity to warfarin and similar metabolite profile (Fig. 1), but with much faster metabolism. Intrinsic clearance measurements for warfarin were used to evaluate the extent to which alginate bead cultures maintain metabolic competence over long timeframes, while $\mathrm{CL}_{\text {int }}$ measurements for coumarin were used to evaluate how encapsulation affected metabolic enzyme phenotype and metabolic competence in primary hepatocytes in the short term. Then, XME expression levels were measured as a way to verify/characterize metabolic competence and its change over time in these culture conditions. Direct comparisons to suspension cultures that had been maintained over the same time frames (days to weeks) were obviously not possible because suspension cultures of primary hepatocytes maintain viability on the order of hours. Thus, the fact that we have measurable enzyme expression 28 days after receiving the cells is a major improvement compared to primary cell suspension, currently regarded as the "gold standard."

Warfarin is predominately metabolized by CYP2C9, whose activity is preserved over long times in these beads. Figure 4 shows that between $50 \%$ and $70 \%$ of the expression of CYP2C9 is found immediately postencapsulation, while our time course study in Figure 5 shows that its expression rebounds nearly to $100 \%$ of suspension culture after 7 days, second (in the group we tested) only to CES1, and best among the CYPs. The high expression of CYP2C9 provides confidence that the $\mathrm{CL}_{\text {int }}$ values derived reflect those we would expect if traditional suspension cultures were able to maintain viability over longer timeframes. The results shown in Supplementary Figure S4 confirm that the typical incubation time of 4 hours used in suspension culture is insufficient for measuring $\mathrm{CL}_{\text {int }}$ for slow clearance chemicals such as $S$-warfarin, as no decrease in parent concentration is detected. The estimated in vivo $\mathrm{CL}_{\text {int }}$ for warfarin based on the alginate bead culture data was within fourfold of the reported in vivo data. ${ }^{23}$
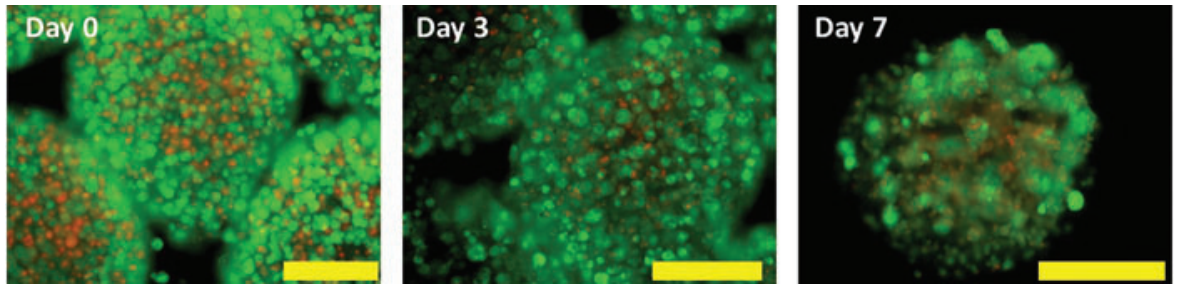

FIG. 6. Images of the alginate beads prepared from the second donor (HUM4179) on day 0 (day of encapsulation, left), day 3 (center), and day 7 (right) using AO/PI staining. AO (green) is a membrane-permeable dye that stains all cells, while PI (orange) is a membrane-impermeable dye that stains only nonviable cells. Yellow scale bars are $250 \mu \mathrm{m}$. AO, acridine orange; PI, propidium iodide. Color images available online at www.liebertpub.com/aivt 


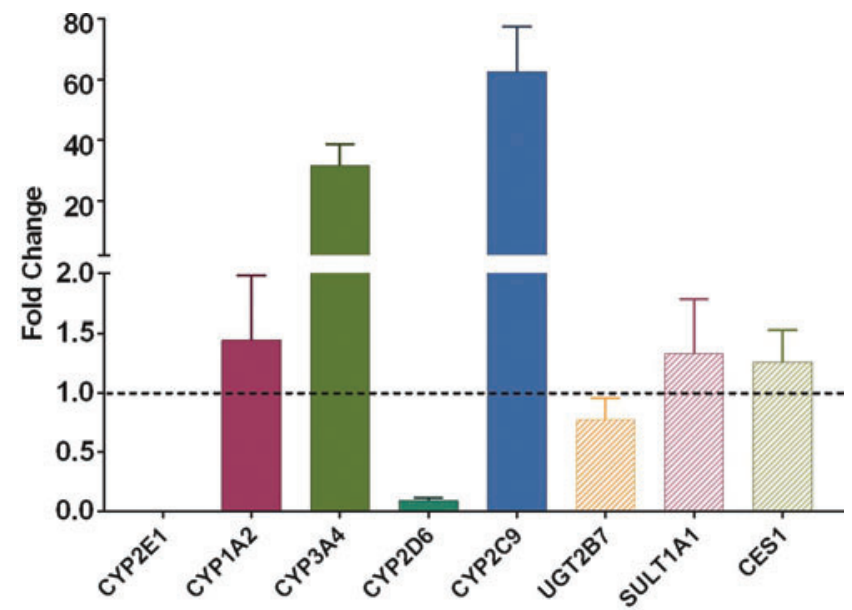

FIG. 7. Gene expression for the third donor (HUM4108) on day 28 of cell culture normalized to expression in beads on day 0. CES is carboxylesterase I, a non-CYP Phase I metabolizing enzyme. Error bars represent the standard deviation in qRT-PCR replicates $(n=3)$. Color images available online at www.liebertpub.com/aivt

Given the large interindividual variability in human metabolism, especially in CYP2C9 that is largely responsible for $S$-warfarin metabolism, our results show promise that this in vitro system can increase accuracy in metabolic clearance estimation for poorly metabolized chemicals.

Although metabolic competence was not compromised immediately after encapsulation, XME expression levels rapidly declined in the first 3 days or so, returning to their preencapsulation levels by 7 days. This suggests that our 7-day incubation period included that window of low metabolic competence during which cells are recovering their functionality, including metabolic competence after encapsulation. Therefore, it is possible that this could have affected and underestimated the $\mathrm{CL}_{\mathrm{int}}$ for warfarin in our study. Some of the evaluated XMEs have not recovered to a level comparable to isolated hepatocytes, including CYP2E1, which is known to play an important role in metabolic activation of a number of chemicals. In addition to the need to increase confidence in $\mathrm{CL}_{\text {int }}$ estimation, an improved metabolic system is required to investigate the effect of metabolite effects, which is often an issue with prolonged exposure to both rapidly and slowly metabolized compounds. Further refinement of culture conditions to specifically improve the relative expression of XMEs among different enzymes, in- cluding those shown to be less successful in this study up to a level comparable to the in vivo condition would be critical to increase the utility of the alginate bead culture in chemical safety assessment with confidence.

The images in Figures 6 and 8 show that the human hepatocytes are dispersed throughout the alginate on day 0 , while by day 3 (Fig. 6) and day 7 (Figs. 6 and 8) aggregates appear. Previous studies with alginate-encapsulated hepatocyte cultures have also shown that aggregation begins in earnest by day 2 , and metabolism rebounds on day 3, peaking on day 7. ${ }^{27-29}$ Hepatocyte aggregation is a common effect on matrices such as Matrigel and also occur in alginate, creating organoid-type structures that display polarity and biliary markers between hepatocytes. Similar to these studies, our results show gene expression increases from day 3 to 7 in culture. Hepatocyte aggregation in alginate is dependent on seeding density, requiring $\geq 5.7 \times 10^{6}$ cells $/ \mathrm{mL}$ alginate. ${ }^{27}$ For comparison, a 1:1.25 (v/v) ratio of cells to $2 \%(\mathrm{w} / \mathrm{v})$ alginate was used in this study-at $\sim 70 \times 10^{6}$ cells $/ \mathrm{mL}$ of pelleted human hepatocytes after 5 minutes at $70 \mathrm{~g}$ centrifugation, this is $\sim 31 \times 10^{6}$ cells/mL alginate, well above what is required for aggregation to take place.

Overall, our results indicated that alginate beads in an improved dynamic culture condition demonstrate great potential as a long-term culture model for slow clearance investigation. Other 3D and/or organotypic cell culture systems, often combined with advanced cell culture devices such as microfluidicbased organ-on-a-chip tools, can maintain viability over long periods with metabolic competence maintained. However, they are not suited for metabolism investigation or kinetic studies in general. For microfluidics, the main issues are difficulties in interpretation of the data for IVIVE, substantial nonspecific binding to large surface areas of tubing and other connections, and most importantly, a large media:cell volume ratio leading to challenges on the analytical side in addition to biological relevance of the incubation conditions. In addition, their fixed geometry means that they are not easily scalable or adaptable to different purposes, for example, rapid screening versus comprehensive recapitulation of in vivo metabolic pathways.

Application of organotypic culture systems such as liver spheroids to metabolism studies has some practical challenges as they are not easily scalable (e.g., one spheroid per well is typical, limited by technical considerations such as aggregation of spheroids) and/or reproducible to consistent size and density. Alginate bead cultures have demonstrated long-term viability and show promise for sustained metabolic competence, they are scalable by adding more or
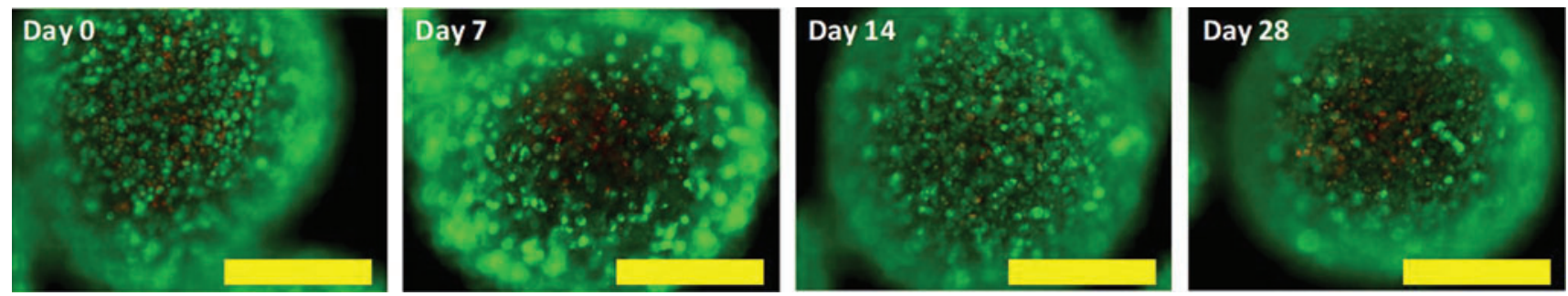

FIG. 8. Images of the beads prepared from the third donor (HUM4108) on day 0 (day of encapsulation, far left), day 7 (center left), day 14 (center right), and day 28 (far right). Yellow scale bars are $400 \mu \mathrm{m}$. Color images available online at www.liebertpub.com/aivt 
fewer beads per well without the issue of aggregation or necrotic cores with more desirable reproducibility in size and cell density of the individual beads.

Despite these potential advantages, however, if they are to be used in traditional culture plates, in vitro kinetic factors become major issues in conducting IVIVE of the results from bead culture. This difficulty arises from the need to exchange a portion of the media each day to ensure sufficient supply of nutrients to the cells, which becomes critical in long-term cultures. Combining the described alginate bead culture in this study and a flow-based culture system can ameliorate this concern, since a single reservoir could be used to continuously supply cells with oxygen and nutrients with media changes reduced to once per week, or even less often. The successful design and implementation of such a system could revolutionize $\mathrm{CL}_{\text {int }}$ experiments for currently challenging compounds. In addition, such a system could be used for repeated dose studies with the advantage of realistic exposure profiles of in vivo parent and metabolites, but generated in an in vitro format.

\section{Conclusions}

We have developed a simple 3D primary hepatocyte culture method that is viable over longer time periods to meet the needs for the reliable measurement of $\mathrm{CL}_{\text {int }}$ for slowly cleared chemicals. Coupled with computational tools for IVIVE, the improved in vitro metabolism tools presented here have potential to address current challenges in in vitrobased safety assessment for persistent chemicals and provide critical data to understand their associated long-term effects. Once accumulated, human metabolism data generated from this in vitro metabolism system can contribute to expanding the domain of applicability of computational models to predict human metabolism, particularly for human-relevant data on clearance and metabolite profiles of lipophilic and/or slowly metabolized chemicals.

\section{Acknowledgments}

This research was funded by the Long-Range Research Initiative of the American Chemistry Council. The authors thank Dr. Andrey Tikunov for assisting with the preparation of the alginate beads.

\section{Author Disclosure Statement}

No competing financial interests exist.

\section{References}

1. NRC. Toxicity Testing in the 21st Century: A Vision and a Strategy. Washington, D.C.: The National Academies Press; 2007.

2. Yoon M, Efremenko A, Blaauboer BJ, et al. Evaluation of simple in vitro to in vivo extrapolation approaches for environmental compounds. Toxicol In Vitro 2014:28;164-170.

3. Jaroch K, Jaroch A, Bojko B. Cell cultures in drug discovery and development: the need of reliable in vitro-in vivo extrapolation for pharmacodynamics and pharmacokinetics assessment. J Pharm Biomed Anal 2018:147;296-312.

4. Sipes NS, Wambaugh JF, Pearce R, et al. An intuitive approach for predicting potential human health risk with the Tox 21 10k library. Environ Sci Technol 2017:51;10786-10796.
5. Clewell RA, McMullen PD, Adeleye Y, et al. Pathway based toxicology and fit-for-purpose assays. Adv Exp Med Biol 2016:856;205-230.

6. Yoon M, Campbell JL, Andersen ME, et al. Quantitative in vitro to in vivo extrapolation of cell-based toxicity assay results. Crit Rev Toxicol 2012:42;633-652.

7. Hakala KS, Suchanova B, Luukkanen L, et al. Rapid simultaneous determination of metabolic clearance of multiple compounds catalyzed in vitro by recombinant human UDPglucuronosyltransferases. Anal Biochem 2005:341;105-112.

8. Rotroff DM, Wetmore BA, Dix DJ, et al. Incorporating human dosimetry and exposure into high-throughput in vitro toxicity screening. Toxicol Sci 2010:117;348-358.

9. Wetmore BA, Wambaugh JF, Ferguson SS, et al. Integration of dosimetry, exposure, and high-throughput screening data in chemical toxicity assessment. Toxicol Sci 2012:125; 157-174.

10. Houston JB, Galetin A. Methods for predicting in vivo pharmacokinetics using data from in vitro assays. Curr Drug Metab 2008:9;940-951.

11. Riley RJ, McGinnity DF, Austin RP. A unified model for predicting human hepatic, metabolic clearance from in vitro intrinsic clearance data in hepatocytes and microsomes. Drug Metab Dispos 2005:33;1304-1311.

12. Obach RS. Prediction of human clearance of twenty-nine drugs from hepatic microsomal intrinsic clearance data: an examination of in vitro half-life approach and nonspecific binding to microsomes. Drug Metab Dispos 1999:27; 1350-1359.

13. Di L, Keefer C, Scott DO, et al. Mechanistic insights from comparing intrinsic clearance values between human liver microsomes and hepatocytes to guide drug design. Eur J Med Chem 2012:57;441-448.

14. Di L, Obach RS. Addressing the challenges of low clearance in drug research. AAPS J 2015:17;352-357.

15. Ballard TE, Wang S, Cox LM, et al. Application of a micropatterned cocultured hepatocyte system to predict preclinical and human-specific drug metabolism. Drug Metab Dispos 2016:44;172-179.

16. Vinci B, Cavallone D, Vozzi G, et al. In vitro liver model using microfabricated scaffolds in a modular bioreactor. Biotechnol J 2010:5;232-241.

17. Dash A, Simmers MB, Deering TG, et al. Hemodynamic flow improves rat hepatocyte morphology, function, and metabolic activity in vitro. Am J Physiol Cell Physiol 2013:304;C1053-C1063.

18. Ukairo O, Kanchagar C, Moore A, et al. Long-term stability of primary rat hepatocytes in micropatterned cocultures. J Biochem Mol Toxicol 2013:27;204-212.

19. Enders JR, McIntire GL. A dilute-and-shoot LC-MS method for quantitating opioids in oral fluid. J Anal Toxicol 2015: 39;662-667.

20. Barter ZE, Bayliss MK, Beaune PH, et al. Scaling factors for the extrapolation of in vivo metabolic drug clearance from in vitro data: reaching a consensus on values of human microsomal protein and hepatocellularity per gram of liver. Curr Drug Metab 2007:8;33-45.

21. Huang Z, Li H, Zhang Q, et al. Characterization of preclinical in vitro and in vivo pharmacokinetics properties for KBP-7018, a new tyrosine kinase inhibitor candidate for treatment of idiopathic pulmonary fibrosis. Drug Des Dev Ther 2015:9;4319-4328.

22. Jigorel E, Houston J B. Utility of drug depletion-time profiles in isolated hepatocytes for accessing hepatic uptake 
clearance: identifying rate-limiting steps and role of passive processes. Drug Metab Dispos 2012:40;1596-1602.

23. Hallifax D, Foster JA, Houston JB. Prediction of human metabolic clearance from in vitro systems: retrospective analysis and prospective view. Pharm Res 2010:27;2150-2161.

24. Pedersen JM, Shim YS, Hans V, et al. Fluid dynamic modeling to support the development of flow-based hepatocyte culture systems for metabolism studies. Front Bioeng Biotechnol 2016:4;72.

25. Varma MV, Steyn SJ, Allerton C, et al. Predicting clearance mechanism in drug discovery: extended clearance classification system (ECCS). Pharm Res 2015:32;37853802.

26. Shaik AN, Bohnert T, Williams DA, et al. Mechanism of drug-drug interactions between warfarin and statins. J Pharm Sci 2016:105;1976-1986.

27. Dvir-Ginzberg M, Gamlieli-Bonshtein I, Agbaria R, et al. Liver tissue engineering within alginate scaffolds: effects of cell-seeding density on hepatocyte viability, morphology, and function. Tissue Eng 2003:9;757-766.

28. Glicklis R, Merchuk JC, Cohen S. Modeling mass transfer in hepatocyte spheroids via cell viability, spheroid size, and hepatocellular functions. Biotechnol Bioeng 2004: 86;672-680.

29. Tikunov AP, Shim YS, Bhattarai N, et al. Dose-response in a high density three-dimensional liver device with real-time bioenergetic and metabolic flux quantification. Toxicol In Vitro 2017:45;119-127.

Address correspondence to: Dr. Miyoung Yoon ToxStrategies, Inc. 1249 Kildaire Farm Road 134 Cary, NC 27511

E-mail: myoon@toxstrategies.com 